\title{
Rotation dependence of a phase delay between plasma edge electron density and temperature fields due to a fast rotating, resonant magnetic perturbation field
}

\author{
H. Stoschus, ${ }^{1}$ O. Schmitz, ${ }^{1}$ H. Frerichs, ${ }^{1}$ M. W. Jakubowski, ${ }^{1,2}$ B. Unterberg, ${ }^{1}$ \\ S. S. Abdullaev, ${ }^{1}$ M. Clever, ${ }^{1}$ J. W. Coenen, ${ }^{1}$ U. Kruezi, ${ }^{1}$ D. Schega, ${ }^{1}$ U. Samm, ${ }^{1}$ \\ and TEXTOR Research Team \\ ${ }^{1}$ Forschungszentrum Jülich GmbH, Institute for Energy Research-Plasma Physics, \\ Association EURATOM-FZJ, Partner in the Trilateral Euregio Cluster, 52425 Jülich, Germany \\ ${ }^{2}$ Max Planck Institute for Plasma Physics, Association IPP-EURATOM, 17491 Greifswald, Germany
}

(Received 26 March 2010; accepted 6 May 2010; published online 15 June 2010)

\begin{abstract}
Measurements of the plasma edge electron density $n_{e}$ and temperature $T_{e}$ fields during application of a fast rotating, resonant magnetic perturbation (RMP) field show a characteristic modulation of both, $n_{e}$ and $T_{e}$ coherent to the rotation frequency of the RMP field. A phase delay $\Phi$ between the $n_{e}(t)$ and $T_{e}(t)$ waveforms is observed and it is demonstrated that this phase delay $\Phi$ is a function of the radius with $\Phi(r)$ depending on the relative rotation of the RMP field and the toroidal plasma rotation. This provides for the first time direct experimental evidence for a rotation dependent damping of the external RMP field in the edge layer of a resistive high-temperature plasma which breaks down at low rotation and high resonant field amplitudes. [doi:10.1063/1.3436614]
\end{abstract}

In magnetically confined high temperature plasmas for exploration of nuclear fusion as future energy source, resonant magnetic perturbation (RMP) fields are a promising tool for control of edge localized modes by fine tuning of the particle and energy exhaust. ${ }^{1-4}$ For the extrapolation of this technique from present experiments toward ITER, ${ }^{5}$ the understanding of RMP field penetration, the resulting perturbed magnetic topology and the plasma transport is of major importance.

The penetration of the external RMP field is determined by plasma response currents due to a nonzero relative rotation frequency $f_{\text {rel }}$ between the highly conductive plasma and the external RMP field. These currents-depending on the framework applied-potentially shield ${ }^{6}$ or $_{\text {amplify }}^{7}$ the external field on resonant magnetic flux surfaces. The actual response current depends in turn on the resistivity of the plasma. At low resistivity, an ideal plasma response with strong shielding currents on the resonant flux surfaces is expected. As resistivity increases, one expects a breakdown of this shielding currents and the opening of magnetic islands. ${ }^{8,9}$ In this paper we study the rotation dependence of the impact of a fast rotating RMP field on the edge electron density $n_{e}(r, t)$ and temperature $T_{e}(r, t)$ fields in a highly resistive (Lunquist number $S=\tau_{R} / \tau_{A} \approx 10^{4}$ with $\tau_{A}$ as Alfvén and $\tau_{R}$ as resistive time) edge plasma. We show in this paper three first time observations, which in combination provide for low RMP field amplitudes $\left(B_{r}<0.2 \mathrm{mT}\right)$ evidence for a shielding of the external field even in the resistive edge of low confinement (L-mode) plasmas at TEXTOR. ${ }^{10}$ The RMP field is generated by a helical RMP coil set, the Dynamic Ergodic Divertor (DED) ${ }^{11}$ generating RMP fields with different toroidal $(n)$ and poloidal $(m)$ base mode numbers of $m / n=12 / 4,6 / 2,3 / 1$, and the capability for RMP field rotation with high frequencies of $\nu_{\mathrm{DED}}=1,2,5$, and $10 \mathrm{kHz}$.

The analysis presented in this letter for high frequency RMP rotation is based on findings on magnetic topology and transport properties during application of static RMP fields. ${ }^{12-14}$ Here, experimental electron density and temperature field reactions were compared with the magnetic topology modeled by field line tracing in vacuum approximation, i.e., the superposition of the vacuum RMP field components onto a stationary axis-symmetric plasma equilibrium. It was shown in summary that a stochastic edge layer is induced, which consists of three different topological domains with different transport properties. On the first inner resonant surface with sufficient RMP field amplitude, the perturbed edge layer is determined by magnetic islands, which can act as convective cells enhancing the transport. ${ }^{4,15}$ Further out, the resonant islands overlap and form a region filled by stochastic field lines leading to an increased radial transport. ${ }^{16}$ The outermost region is formed as a new three-dimensional (3D) scrape off layer (SOL) by coherent magnetic field lines with short connection length $L_{c}$ to the wall bundled into so-called laminar magnetic flux tubes. They are embedded into a sea of stochastic field lines and termed laminar zone. It was revealed in Refs. 13 and 14 that laminar flux tubes impose a poloidal modulation to electron density $n_{e}$ and temperature $T_{e}$ fields. In this paper we demonstrate for the first time that this poloidal modulation is preserved and rotates locked to a fast rotating RMP field but that a pronounced phase delay $\Phi$ between $n_{e}$ and $T_{e}$ exists, which in fact is a function of the radius. Further more $\Phi$ is shown to vanish with increasing RMP amplitude for low relative rotation frequency between the RMP field and the plasma fluid only while being maintained for high relative rotation.

The experiments were performed with a poloidal/ toroidal mode number $m / n=3 / 1$ RMP field, a low order electro magnetic multipole and therefore high resonant field amplitudes in the plasma edge layer. We analyze a highly resistive ohmic plasma with circular shape and limited on the DED target. Two opposite RMP rotation frequencies of $\nu_{\mathrm{DED}}= \pm 974 \mathrm{~Hz}$ were used, where the sign of the rotation 

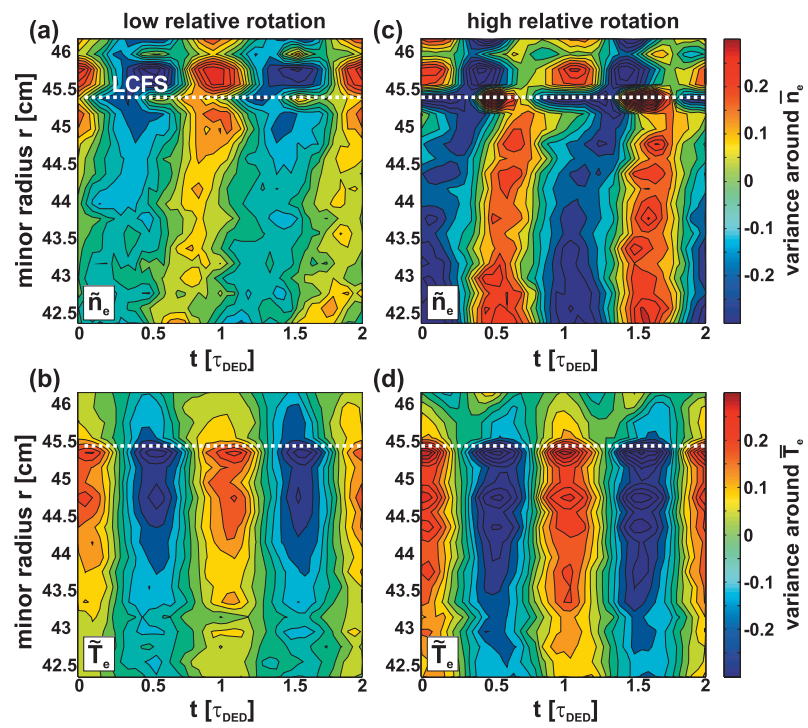

FIG. 1. (Color) Normalized electron density (upper row) and electron temperature (lower row) as contour plot vs minor radius and time (normalized to DED rotation period $\left.\tau_{\text {DED }}\right)$ for low relative rotation $\left(f_{\text {rel }}=+0.2 \mathrm{kHz}\right.$, left column, \#104741) and high relative rotation $\left(f_{\text {rel }}=+1.8 \mathrm{kHz}\right.$, right column, \#104739).

direction is defined in the direction of the electron diamagnetic drift direction $\vec{\omega}_{\text {ed }}$. This yields two rotation cases: low relative rotation will refer to RMP field rotation parallel to $\vec{\omega}_{\text {ed }}$ and hence a relative rotation frequency of $f_{\text {rel }}$ $=+0.2 \mathrm{kHz}$. High relative rotation will refer to RMP rotation in opposite direction with $f_{\text {rel }}=+1.8 \mathrm{kHz}$. The global plasma parameters in the core/edge are as follows: electron density $n_{e}(0)=2 \cdot 10^{19} \mathrm{~m}^{-3}, \quad n_{e}(r / a=0.92)=8 \cdot 10^{18} \mathrm{~m}^{-3}$, electron temperature $T_{e}(0)=1 \mathrm{keV}$, and $T_{e}(r / a=0.92)=60 \mathrm{eV}$. All global parameters stayed constant during the RMP field application with an amplitude of $I_{\mathrm{DED}}=1 \mathrm{kA}$. No changes in the global plasma parameters and in the MHD spectrum, e.g., no indications for an $n=1$ tearing mode ${ }^{17}$ were observed. It is important to note that the toroidal rotation frequency increases linearly with the RMP strength up to $\Omega_{\text {plasma,tor }}$ $=v_{\text {tor }} / R=+5 \mathrm{kHz}$ for both RMP rotation directions. This is compatible with the finding with static RMP field that the RMP amplitude drives the toroidal rotation due to an open, stochastic edge layer causing additional electron losses which are balanced by a radial ion current. This current causes an increase of the toroidal rotation by $\vec{j} \times \vec{B}_{\text {toroidal }}$ forces ${ }^{18}$ and seems to exist as well under the impact of rotating RMP fields. The $n_{e}(r, t)$ and $T_{e}(r, t)$ fields within $r / a$ $=0.9-1.05$ are measured by a supersonic helium $(\mathrm{SHE})^{19}$ beam diagnostic based on emission spectroscopy on thermal helium. ${ }^{20}$ Due to a low beam divergence $\left(\Varangle \pm 1^{\circ}\right)$ and a high helium density in the narrow beam, $n_{e}$ and $T_{e}$ can be measured very localized with high radial $(\Delta r=2 \mathrm{~mm})$ and high time resolution $(\Delta t=25 \mu \mathrm{s})$.

Figure 1 shows the normalized electron density $\widetilde{n}_{e}$ and temperature $\widetilde{T}_{e}$ fields obtained with the SHE diagnostic. Both quantities are normalized to its mean values $\bar{n}_{e}$ and $\bar{T}_{e}$ : $\tilde{n}_{e}=\left(n_{e}-\bar{n}_{e}\right) / \bar{n}_{e}$ and $\tilde{T}_{e}=\left(T_{e}-\bar{T}_{e}\right) / \bar{T}_{e}$. The DED period $\tau_{\mathrm{DED}}=1 / \nu_{\mathrm{DED}}$ is employed as time axis and data are shown for two DED periods. These measurements show for the first
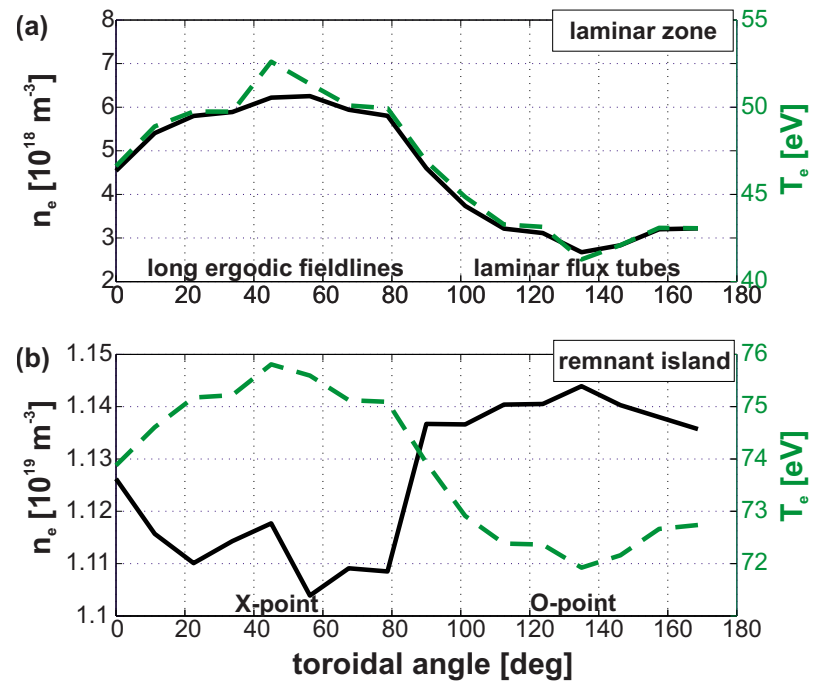

FIG. 2. (Color online) Toroidal profiles of $n_{e}$ (black/solid line, left axis) and $T_{e}$ (green/dashed line, right axis) modeled with EMC3-EIRENE in vacuum approximation through the laminar zone (a) and through a remnant island chain (b) in $m / n=6 / 2$ base mode configuration.

time a modulation of $\widetilde{n}_{e}$ and $\widetilde{T}_{e}$ induced by the rotating RMP field showing that the plasma edge structure revealed for static RMP is preserved but rotates now with the RMP rotation frequency. However, we will show that there is a clear impact of the rotation on the modulation suggesting a dependence of the magnetic topology on the relative rotation level. Figures 1(a) and 1(b) show $\widetilde{n}_{e}$ and $\widetilde{T}_{e}$ for the case with low relative rotation. We observe a clear modulation of $\widetilde{n}_{e}$ and $\widetilde{T}_{e}$ coherent to the DED period and we detect that $\widetilde{n}_{e}$ and $\widetilde{T}_{e}$ increase simultaneously at $t=1 \cdot \tau_{\text {DED }}$ without any phase delay $\Phi$ between $n_{e}$ and $T_{e}$. In contrast, the high relative rotation case shown in Figs. 1(c) and 1(d) reveals a clear shift of the $\tilde{n}_{e}$ maximum toward $t=0.5 \cdot \tau_{\mathrm{DED}}$. This transfers to a phase delay between $\widetilde{n}_{e}$ and $\widetilde{T}_{e}$ of $\Phi \approx 180^{\circ}$ at a radius of $r=42.5 \mathrm{~cm}$.

In order to approach explanation of this phase delay we compare to the fluid plasma and kinetic neutral transport code package EMC3-Eirene. ${ }^{21,22}$ This code employs a threedimensional grid including the static magnetic topology in vacuum approximation. The numerical analysis of a similar discharge showed that the phase $\Phi$ between the $n_{e}$ and $T_{e}$ waveforms of a toroidal cut depends on the actual topological domain of the cut. With this attempt we assume a rotation of a magnetic topology similar to the static case without field screening. Figure 2 depicts a modulation in $n_{e}(\phi)$ and $T_{e}(\phi)$ in the toroidal direction $\phi$. The toroidal profile at the radial position of the laminar zone [Fig. 2(a)] shows a simultaneous drop in $n_{e}$ and $T_{e}$ and hence a phase $\Phi \rightarrow 0$. In contrast, a counter modulation $(\Phi \rightarrow \pi)$ at the location of a remnant island chain [Fig. 2(b)] is seen, which stems from heat transport around the island across the X-point and a local confinement of particles at the O-point. In addition, functionality of such magnetic structures as convective cells is capable to impose poloidally modulating $n_{e}$ and $T_{e}$ fields, too. ${ }^{4,15}$ Based on this numerical result, we use for the interpretation of the measurement the phase delay $\Phi$ as an indicator for the locally dominant topological domain and define $\Phi \rightarrow 0$ as lami- 


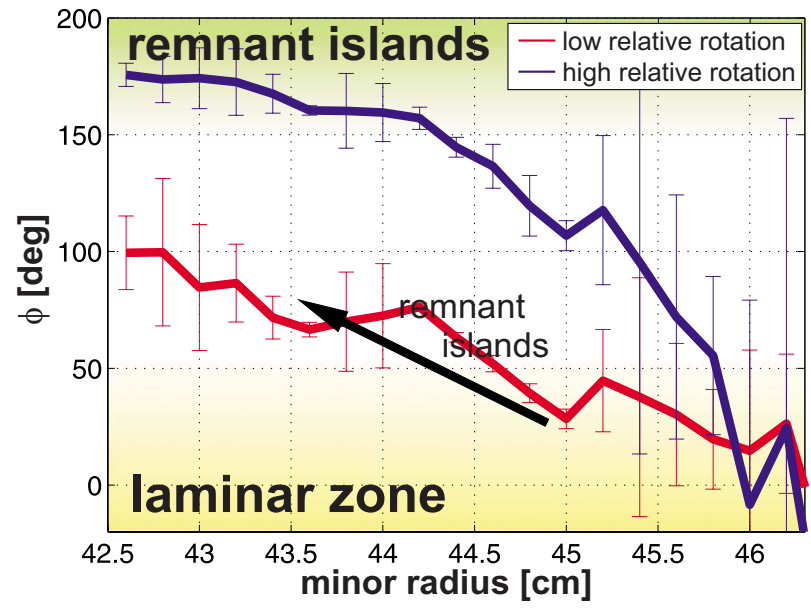

FIG. 3. (Color online) Phase $\Phi$ between $n_{e}$ and $T_{e}$ as a function of the minor radius for low relative rotation $\left(f_{\text {rel }}=+0.2 \mathrm{kHz}, \# 104741\right.$, red/light line $)$ and high relative rotation $\left(f_{\text {rel }}=+1.8 \mathrm{kHz}, \# 104739\right.$, blue/dark line $)$.

nar zone and $\Phi \rightarrow \pi$ as a region with dominant remnant islands.

A clear radial dependence of $\Phi(r)$ was observed experimentally, as shown in Fig. 3 and the gradient of $\Phi(r)$ exhibits a rotation dependence. For both relative rotation levels, a phase $\Phi \approx 0$ is observed for $r>46 \mathrm{~cm}$, where the laminar zone is present in modeling and evidently formed also in the experiment. For the low relative rotation case, $\Phi(r)$ increases up to $\Phi(r)=100^{\circ}$ at $r=42.5 \mathrm{~cm}$ indicating that the magnetic topology in this radial direction changes from a SOL laminar flux tube layer toward remnant islands as inner boundary of the stochastic edge layer. However, increasing the relative rotation leads to a stronger gradient of $\Phi(r)$ which provides evidence that here the magnetic islands are still preserved, not yet broken up and therefore not transformed into laminar flux tubes as occurring for low relative rotation at this radial position. In this interpretation, the low relative rotation case is dominated by laminar flux tubes and the high relative rotation case by remnant islands as a prestage of stochastization for lower RMP amplitude.

In order to relate these experimental findings to the magnetic topology, we simulate a potential damping of the RMP field in the plasma by modeling of the perturbed topology in the detection volume in vacuum approach for different RMP currents, as shown in Fig. 4. The perturbed topology for three different perturbation currents $I_{\mathrm{DED}}=0.5,0.75$, and $1.0 \mathrm{kA}$ is shown as color coded contour plot of the field line connection length $L_{c}$ from wall to wall with a Poincaré plot as an overlay. The measurement position of the SHE diagnostic is highlighted gray. We find that with a perturbation as in the experiment, a region with stochastic field lines with a broad laminar zone $\left(L_{c}<3\right.$ poloidal turns) at $45.5<z<47 \mathrm{~cm}$ develops in the observation volume. This shows that at low rotation and comparable high DED amplitude the interpretation of no phase in the laminar zone and an increasing phase for occurrence of remnant islands which was extracted from static 3D fluid modeling provides a reasonable explanation. However, the experimental measurement at higher rotation suggests that the radial domain of the laminar zone shrinks and the remnant island layer seems to

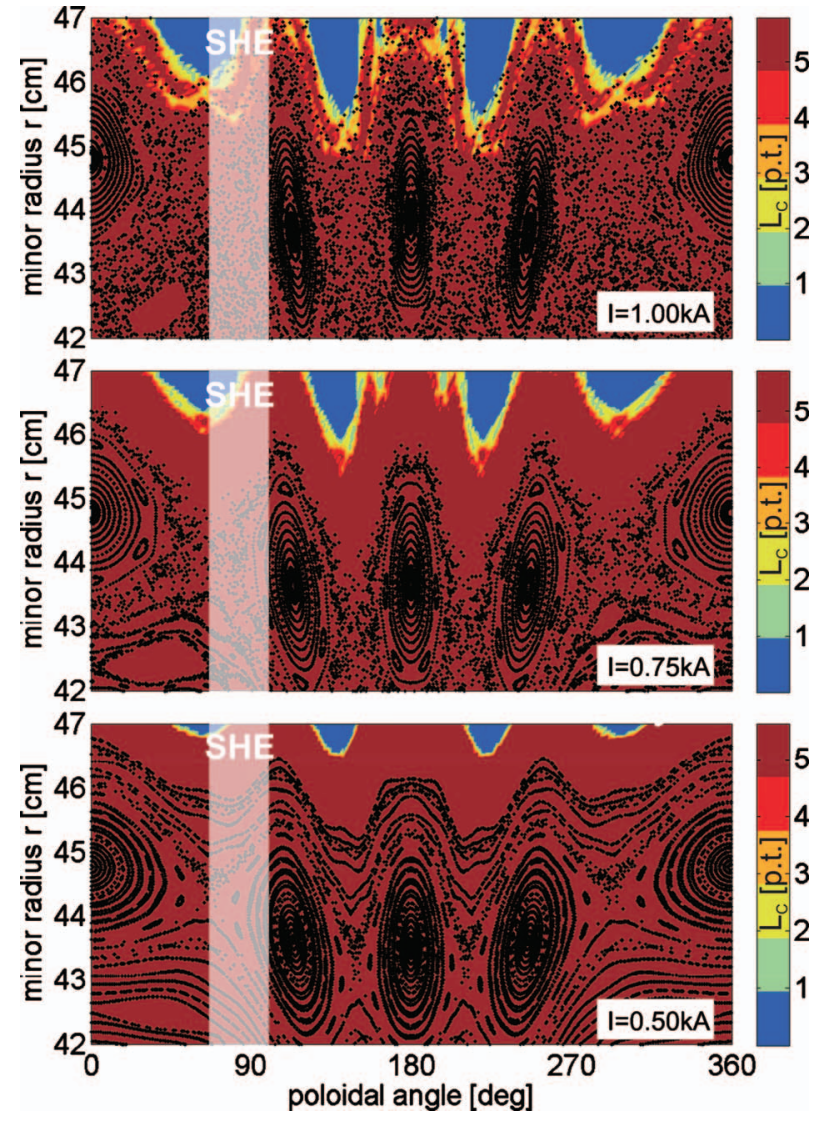

FIG. 4. (Color) Modeled magnetic topology with the ATLAS code (Refs. 12 and 13) in vacuum approximation for different DED currents in coordinates of the minor radius $r$ vs the poloidal angle. A laminar plot is shown with an overlayed Poincaré plot. The position of the SHE diagnostic is highlighted gray.

be extended toward the edge. The same characteristics are seen in the magnetic field line tracing for a globally damped RMP field in reducing the DED current. In this case the resonant islands on this flux surfaces are not yet broken up and accordingly the stochastic region is replaced by remnant islands chains with a decreased radial extension of the laminar zone. This provides for the high rotation case first direct experimental evidence for a damping of the external RMP field inside the plasma edge changing the magnetic topology in such a way that the laminar zone is not generated but magnetic islands are present as expected only for lowered local resonant amplitudes.

This process of net reduction of the external field was recently modeled by means of a four-field drift-fluid model. ${ }^{8}$ Here a break-down of the response currents damping the local resonant components was predicted with increasing perturbation $B_{r}$. This is also found by analysis of the dependence of $\Phi\left(B_{r}\right)$ on the perturbation level and for RMP rotation frequencies of $\nu_{\mathrm{DED}}=+/-974 \mathrm{~Hz}$. The plasma scenario was adjusted with counter neutral beam injection and cocurrent torque input in order to reach a higher toroidal plasma rotation frequency of $\Omega_{\text {tor }}=+6 \mathrm{kHz}$. Figure 5 shows the dependence of $\Phi\left(I_{\mathrm{DED}}\right)$ at the rational flux surface $q=5$ on the DED current $I_{\mathrm{DED}}$ for the low relative rotation $\left(f_{\text {rel }}=5 \mathrm{kHz}\right)$ and the high relative rotation case $\left(f_{\text {rel }}=7 \mathrm{kHz}\right)$. For high relative rotation (blue/dark line), $\Phi$ stays constant at $\Phi=140^{\circ}$ indicating that a magnetic island is 


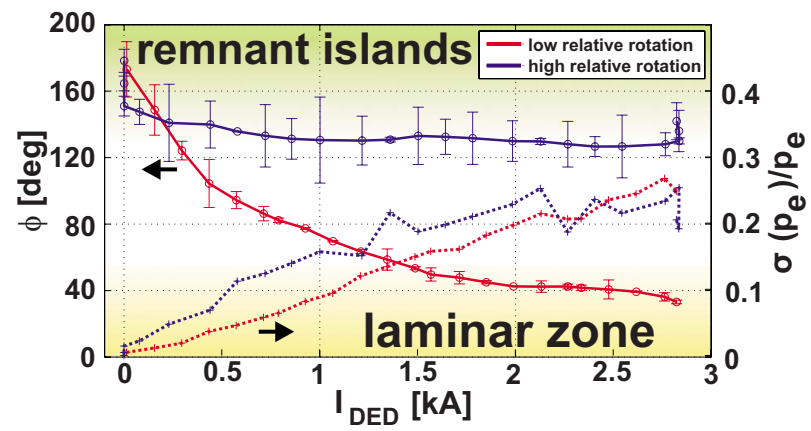

FIG. 5. (Color online) Phase $\Phi$ between $n_{e}$ and $T_{e}$ (solid line, left axis) and modulation depth of electron pressure (dotted line, right axis) as a function of the external RMP current $I_{\text {DED }}$ for low relative rotation $\left(f_{\text {rel }}=5 \mathrm{kHz}\right.$, \#110141-\#110146, red/light line) and high relative rotation $\left(f_{\mathrm{rel}}=7 \mathrm{kHz}\right.$, \#110247-\#110251, blue/dark line) at rational flux surface $q=5$.

present independently of the DED current. In contrast, for low relative rotation (red/light line) $\Phi$ decreases with increasing $I_{\mathrm{DED}}$ from $\Phi=180^{\circ}$ to $\Phi=35^{\circ}$. This provides evidence for breaking up the outermost $q=5$ magnetic island chain forming a laminar zone. At high perturbations $\left(I_{\mathrm{DED}}\right.$ $>1.5 \mathrm{kA}$ ) finally a pure short connection length layer (laminar zone) is developed suggested by a vanishing phase delay. For both RMP rotation scenarios the electron pressure modulation, i.e., the standard deviation $\sigma$ at the RMP rotation frequency normalized to its mean value $\left(\sigma\left(p_{e}\right) / p_{e}\right)$, increases showing rising impact of the topological structures discussed on the plasma edge.

In summary, we show in this letter for the first time measurements of a modulation of $n_{e}$ and $T_{e}$ fields coherent to the rotation frequency of an externally applied RMP field. This modulation proves that a perturbed magnetic boundary layer is imposed even with fast RMP rotations in general. A phase delay $\Phi(r)$ between $n_{e}$ and $T_{e}$ was found as a function of radius $r$ showing that the typical topological domains of a perturbed boundary with static RMP fields can be identified. In particular, a laminar zone with $\Phi \rightarrow 0$ at the edge exists and remnant islands with $\Phi \rightarrow \pi$ toward smaller radii. However, occurrence of either domain depends on the relative rotation between the toroidal plasma rotation and RMP field. The radial gradient $\nabla \Phi(r)$ steepens with increasing rotation which shows that the stochastic layer found at low rotation is replaced by remnant magnetic islands at higher relative rotation. A reduced phase $\Phi$ is measured with increasing perturbation current which provides in case of low rotation and high resonant field amplitude evidence for the transition to a topology which agrees with the vacuum magnetic modeling prediction. However, for higher rotation and/or lower resonant field amplitude, an increasing phase is measured. These observations provide direct experimental evidence that shielding of the external field takes place within this highly resistive TEXTOR edge plasma for (a) low rotation and small RMP amplitude and (b) high rotation at any perturbation strength accessible in the actual experiment. As this interpretation relies so far on $n_{e}$ and $T_{e}$ measurement and vacuum based topology and transport modeling only, it is important to include in future also at least radial electrical field measurements and comparisons to more complete numerical models such as Refs. 4, 8, and 15 for a complete understanding of the underlying RMP penetration.
Supported by the German Research Foundation (DFG) under Grant Nos. UN 265/1-1 and GRK 1203.

${ }^{1}$ T. E. Evans, R. A. Moyer, K. H. Burrell, M. E. Fenstermacher, I. Joseph, A. W. Leonard, T. H. Osborne, G. D. Porter, M. J. Schaffer, P. B. Snyder, P. R. Thomas, J. G. Watkins, and W. P. West, Nat. Phys. 2, 419 (2006).

${ }^{2}$ Y. Liang, H. R. Koslowski, P. R. Thomas, E. Nardon, B. Alper, P. Andrew, Y. Andrew, G. Arnoux, Y. Baranov, M. Bécoulet, M. Beurskens, T. Biewer, M. Bigi, K. Crombe, E. De La Luna, P. de Vries, W. Fundamenski, S. Gerasimov, C. Giroud, M. P. Gryaznevich, N. Hawkes, S. Hotchin, D. Howell, S. Jachmich, V. Kiptily, L. Moreira, V. Parail, S. D. Pinches, E. Rachlew, and O. Zimmermann, Phys. Rev. Lett. 98, 265004 (2007).

${ }^{3}$ K. H. Finken, T. E. Evans, D. Reiter, K. H. Spatschek, and W. Suttrop, Nucl. Fusion 48, 024001 (2008).

${ }^{4}$ E. Nardon, M. Bécoulet, G. Huysmans, and O. Czarny Phys. Plasmas 14, 092501 (2007).

${ }^{5}$ ITER Physics Basis Editors, ITER Physics Expert Group Chairs, CoChairs, ITER Joint Central Team, and Physics Integration Unit, Nucl. Fusion 39, 2137 (1999).

${ }^{6}$ A. Cole and R. Fitzpatrick, Phys. Plasmas 13, 032503 (2006).

${ }^{7}$ J. K. Park, M. J. Schaffer, J. E. Menard, and A. H. Boozer, Phys. Rev. Lett. 99, 195003 (2007).

${ }^{8}$ D. Reiser and D. Chandra, Phys. Plasmas 16, 042317 (2009).

${ }^{9}$ R. Fitzpatrick, Phys. Plasmas 11, 3961 (2004).

${ }^{10}$ O. Neubauer, G. Czymek, B. Giesen, P. W. Hüttemann, M. Sauer, W. Schalt, and J. Schruff, Fusion Sci. Technol. 47, 76 (2005).

${ }^{11}$ K. H. Finken, S. S. Abdullaev, A. Kaleck, and G. H. Wolf, Nucl. Fusion 39, 637 (1999).

${ }^{12}$ S. S. Abdullaev, K. H. Finken, M. W. Jakubowski, S. V. Kasilov, M. Kobayashi, D. Reiser, D. Reiter, A. M. Runov, and R. Wolf, Nucl. Fusion 43, 299 (2003).

${ }^{13}$ M. W. Jakubowski, O. Schmitz, S. S. Abdullaev, S. Brezinsek, K. H. Finken, A. Krämer-Flecken, M. Lehnen, U. Samm, K. H. Spatschek, B. Unterberg, and R. C. Wolf (TEXTOR Team), Phys. Rev. Lett. 96, 035004 (2006).

${ }^{14}$ O. Schmitz, M. W. Jakubowski, H. Frerichs, D. Harting, M. Lehnen, B. Unterberg, S. S. Abduallaev, S. Brezinsek, I. Classen, T. Evans, Y. Feng, K. H. Finken, M. Kantor, D. Reiter, U. Samm, B. Schweer, G. Sergienko, G. W. Spakman, M. Tokar, E. Uzgel, R. C. Wolf, and TEXTOR Team, Nucl. Fusion 48, 024009 (2008).

${ }^{15}$ M. Leconte, P. Beyer, X. Garbet, and S. Benkadda, Nucl. Fusion 50, 054008 (2010).

${ }^{16}$ O. Schmitz, T. E. Evans, M. E. Fenstermacher, E. A. Unterberg, M. E. Austin, B. D. Bray, N. H. Brooks, H. Frerichs, M. Groth, M. W. Jakubowski, C. J. Lasnier, M. Lehnen, A. W. Leonard, S. Mordijck, R. A. Moyer, T. H. Osborne, D. Reiter, U. Samm, M. J. Schaffer, B. Unterberg, and W. P. West (DIII-D and TEXTOR Research Teams), Phys. Rev. Lett. 103, 165005 (2009).

${ }^{17}$ H. R. Koslowski, E. Westerhof, M. de Bock, I. Classen, R. Jaspers, Y. Kikuchi, A. Krämer-Flecken, A. Lazaros, Y. Liang, K. Löwenbrück, S. Varshney, M. von Hellermann, R. Wolf, O. Zimmermann, and TEXTOR Team, Plasma Phys. Controlled Fusion 48, B53 (2006).

${ }^{18}$ B. Unterberg, C. Busch, M. de Bock, J. W. Coenen, K. H. Finken, St. Jachmich, M. W. Jakubowski, Y. Kikuchi, A. Krämer-Flecken, M. Lehnen, U. Samm, O. Schmitz, S. Soldatov, M. Z. Tokar, M. von Hellermann, R. C. Wolf, Y. Xu, and TEXTOR Team, J. Nucl. Mater. 363-365, 698 (2007).

${ }^{19}$ O. Schmitz, S. Brezinsek, I. Classen, M. Clever, J. W. Coenen, E. Delabie, K. H. Finken, M. W. Jakubowski, M. Kantor, A. Krämer-Flecken, U. Kruezi, M. Lehnen, Y. Liang, D. Reiter, U. Samm, B. Schweer, G. W. Spakman, H. Stoschus, G. Telesca, B. Unterberg, E. Uzgel, and Y. Xu, AIP Conf. Proc. 993, 135 (2008).

${ }^{20}$ O. Schmitz, I. L. Beigman, L. A. Vainshtein, B. Schweer, M. Kantor, A. Pospieszczyk, Y. Xu, M. Krychowiak, M. Lehnen, U. Samm, B. Unterberg, and TEXTOR Team, Plasma Phys. Controlled Fusion 50, 115004 (2008).

${ }^{21}$ M. Kobayashi, Y. Feng, F. Sardei, D. Reiter, K. H. Finken, and D. Reiser, Nucl. Fusion 44, S64 (2004).

${ }^{22}$ H. Frerichs, D. Reiter, O. Schmitz, T. E. Evans, and Y. Feng, Nucl. Fusion 50, 034004 (2010). 